\title{
APPLE PRODUCTION INVESTMENT OPPORTUNITIES IN GEORGIA AND GLOBAL CONTEXT
}

\section{EKATERINE ZVIADADZE}

Invited Lecturer

Ivane Javakhishvili Tbilisi State University, Georgia

ekaterine74@yahoo.com

\section{LASHA ZIVZIVADZE}

\section{Associate Professor}

Business and Technology University (BTU), Georgia

I.zivzivadze@yahoo.com

\section{MIKHEILI KUCHAVA}

NNLE Rural Development Agency, Georgia

mikheili.Kuchava@rda.gov.ge

Abstract. The main objective of the presented paper is to identify investment opportunities in the apple value chain and also to evaluate the specific apple project using the state programs/projects. The methodological part provides a financial evaluation of a specific business project, taking into account state funding programs/projects. Furthermore, general investment opportunities of apples are analyzed in the paper. In addition, in order to study the investment potential, the investment climate of Georgia and Georgia's international ratings are discussed. A brief description and analysis of various state support projects are also provided. Additionally, an overview of local apples and the world market is given.

Due to the climate conditions and soil types of Georgia, the production of intensive apple varieties has a great potential in the country. Promoting the cultivation of intensive apple orchards through the use of various state supporting programs/projects and full compliance with apple production technologies, Georgia will have potential to increase the average apple yield per hectare. It is important for Georgia to use the existing free trade relations with other countries and to diversify the apple export market, which will further increase its competitiveness.

Based on the financial evaluation, key performance indicators are positive and beneficial for the investors, which should give the motivation to the investors to invest in the apple value chain. In the field of agriculture, the continuation of the implementation of various state co-financing projects/programs and their development will give additional motivation to local and foreign investors, which will further increase the competitiveness of Georgian apple value chain.

KEYWORDS: APPLE PRODUCTION, VALUE CHAIN, INVESTMENT POTENTIAL, ECONOMIC EVALUATION.

For citation: Zviadadze, E., Zivzivadze, \& L., Kuchava, M. (2021). Apple Production Investment Opportunities in Georgia and Global Context. Globalization and Business. 12, 197-205. (In Georgian). https://doi.org/10.35945/gb.2021.12.028 


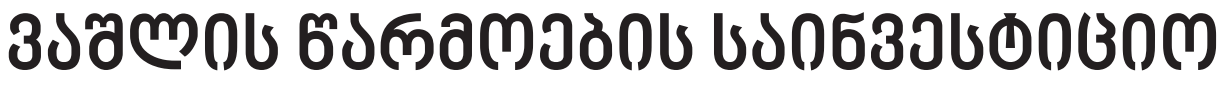

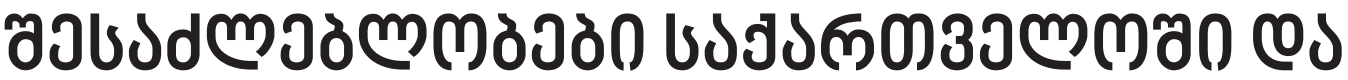

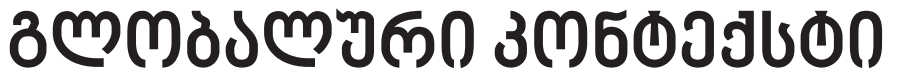

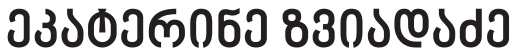

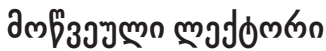

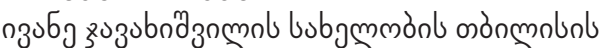

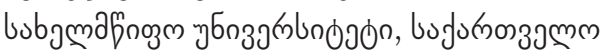 \\ ekaterine74@yahoo.com
}

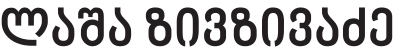

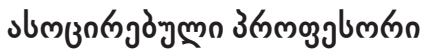

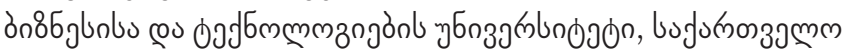

I.zivzivadze@yahoo.com

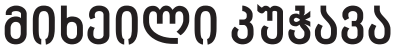

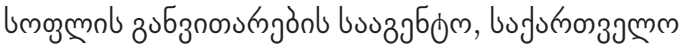

mikheili.Kuchava@rda.gov.ge

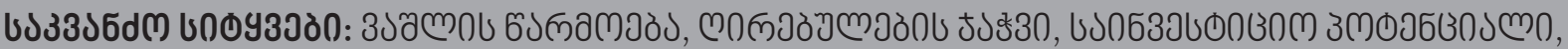

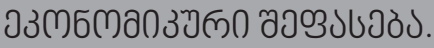

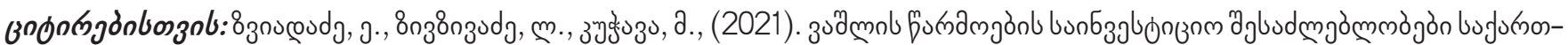

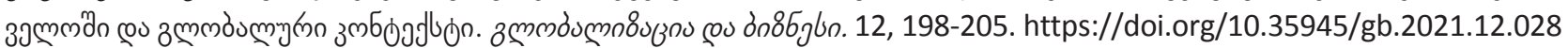

\section{əงเ১З১ल0}

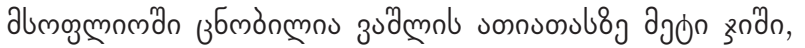

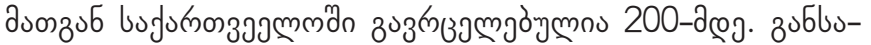

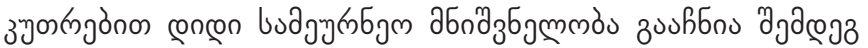

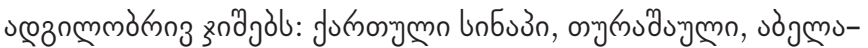

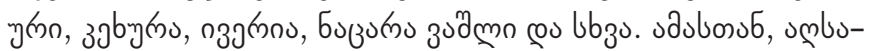

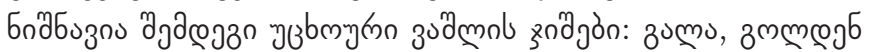

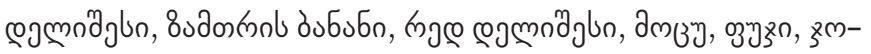

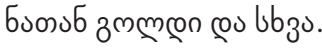

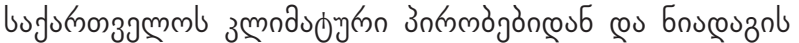

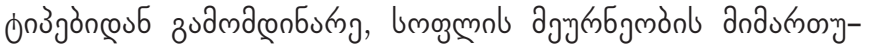

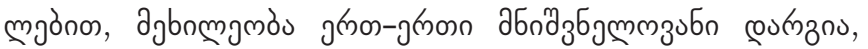

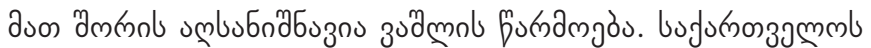

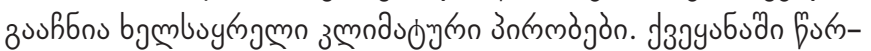

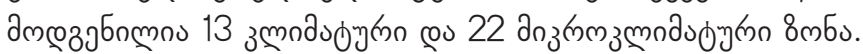

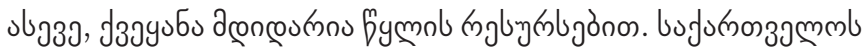

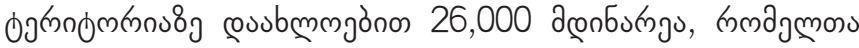

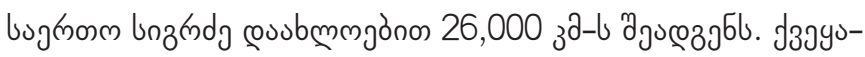

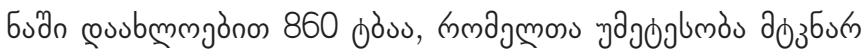
fyumb gоง

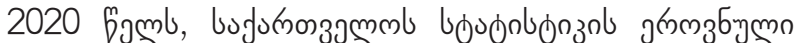

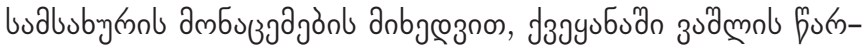

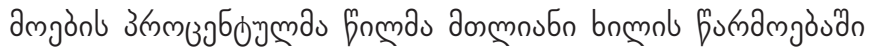

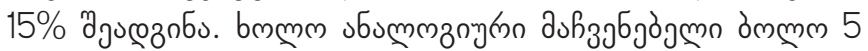

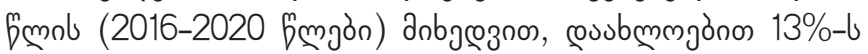
aju@3jol.

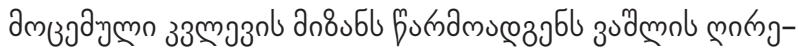

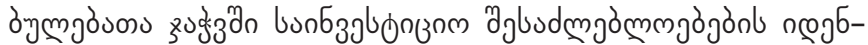

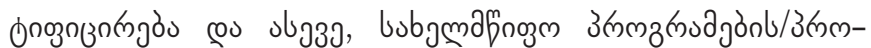

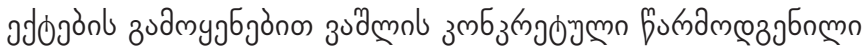

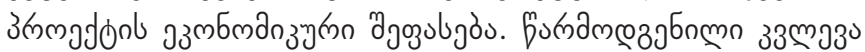

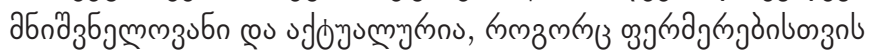

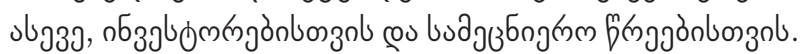

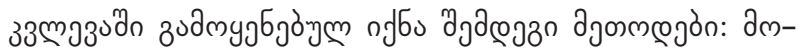

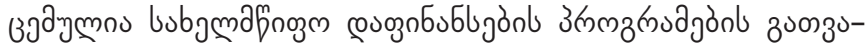




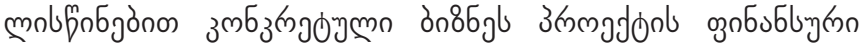

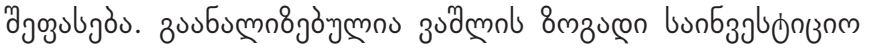

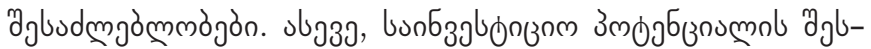

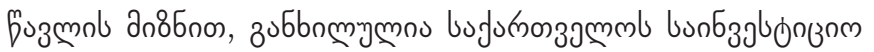

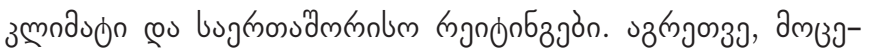

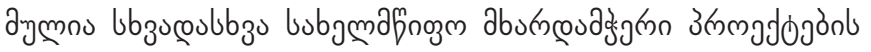

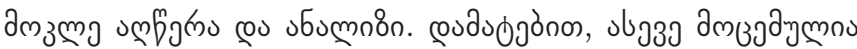

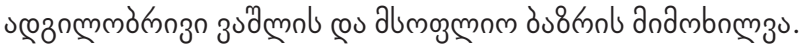

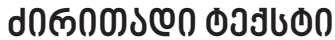

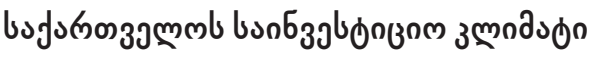

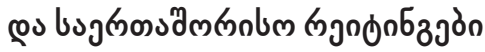

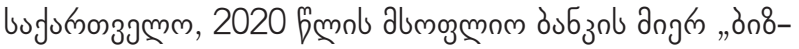

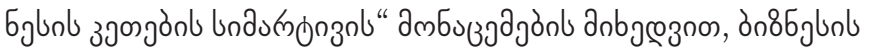

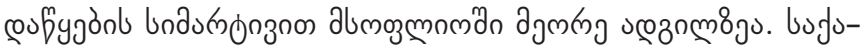

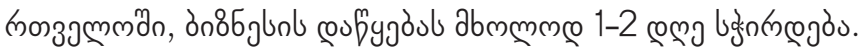

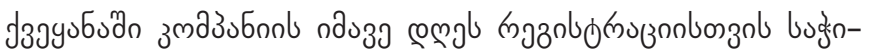

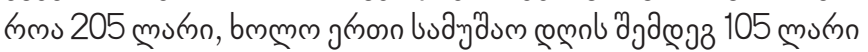
(Business Registration, 2021).

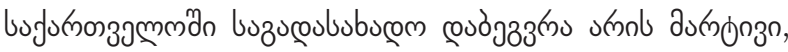

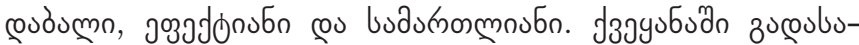
bucgòn sz

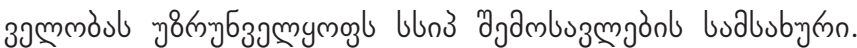

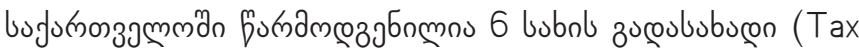

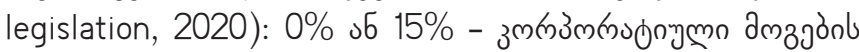

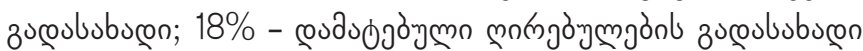

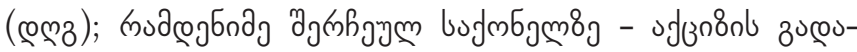
bubucon; 20\% - buagambuzmmm zucoububucon; 0\%, 5\% s6 12\%

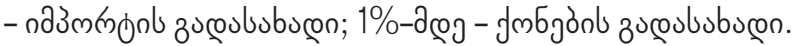

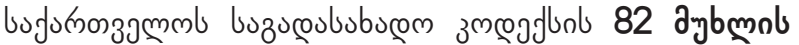

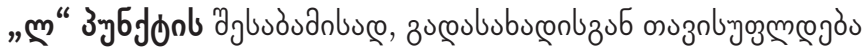

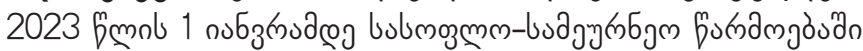

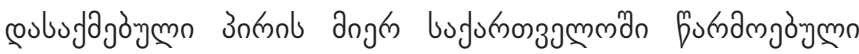

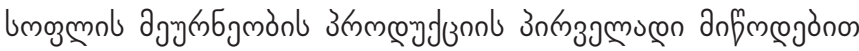

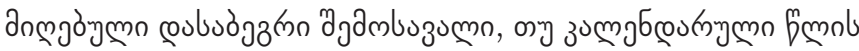

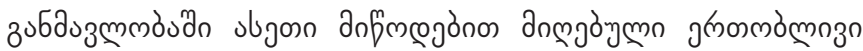

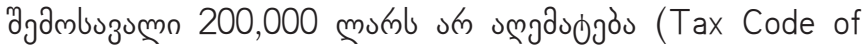
Georgia, 2021).

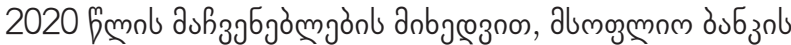

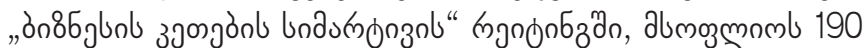

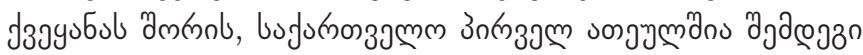
змдзмбјбблабоn

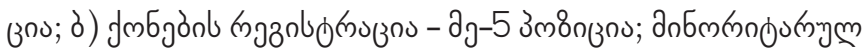

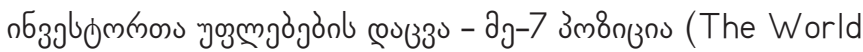
Bank Group, 2020).

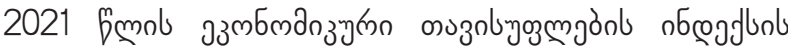

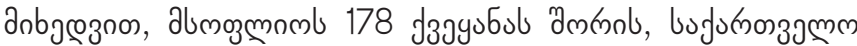

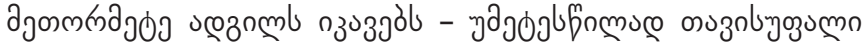
उзmbmanzo (Index of Economic Freedom, 2021).

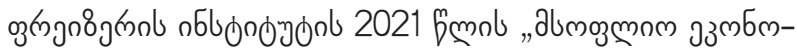

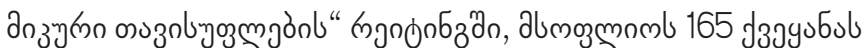

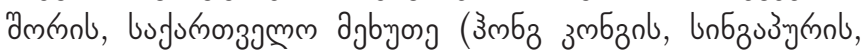

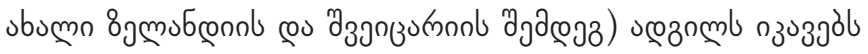
(Fraser Institute, 2021).

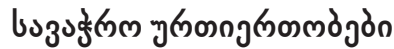

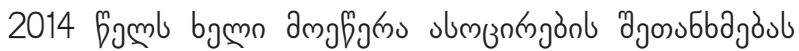

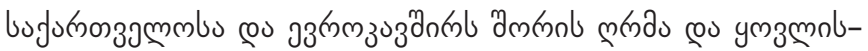

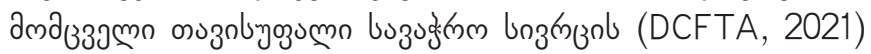
gybubgó.

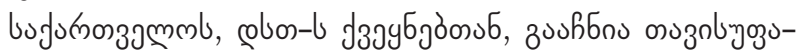

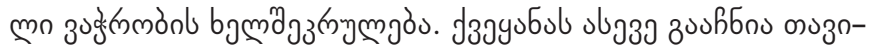

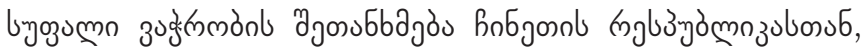

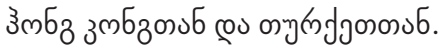

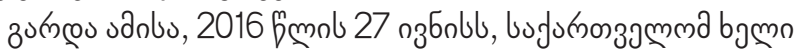

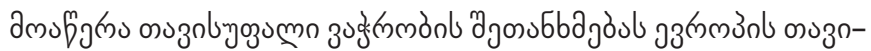

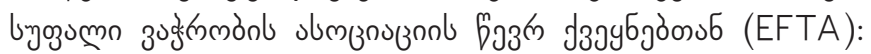

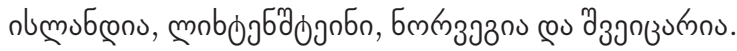

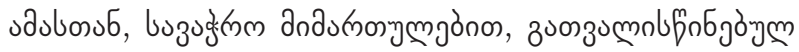

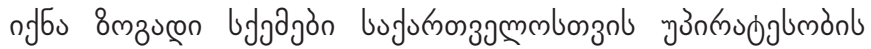

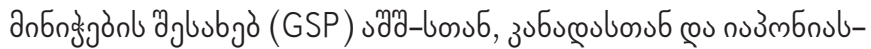

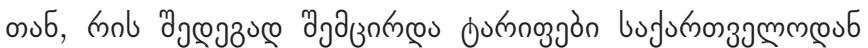

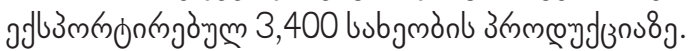

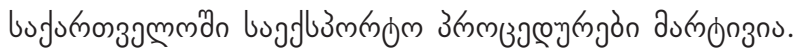

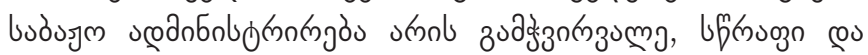

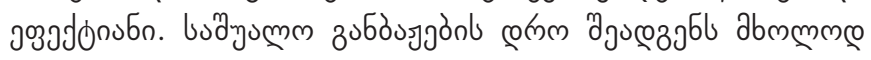
15-20 Gyorb (New Customs Code, 2021).

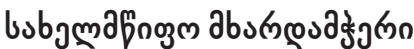

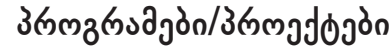

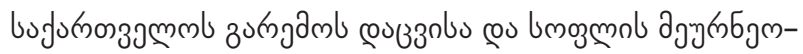
onl buanбonb

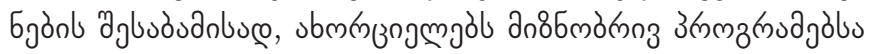

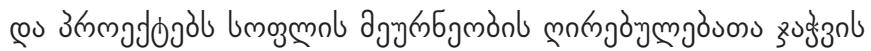

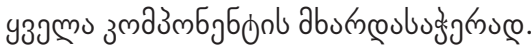

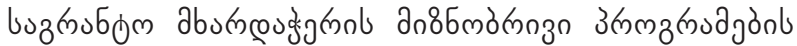

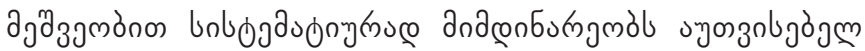

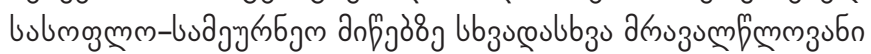

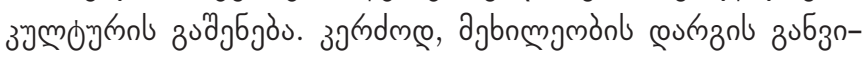

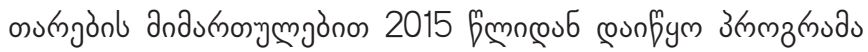

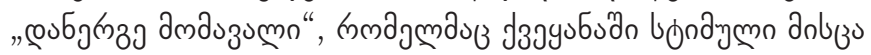

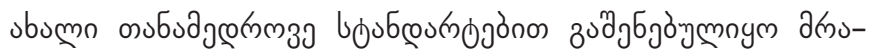

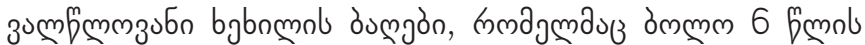

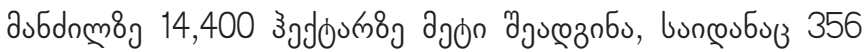

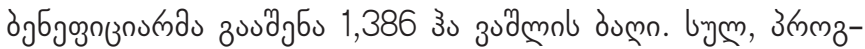




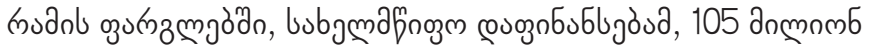
mumb zucouogutrobs („Plant the Future” Project, 2021).

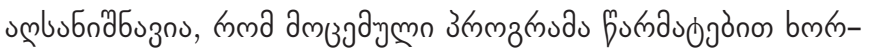

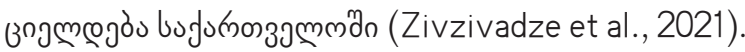

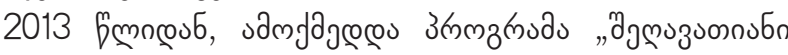

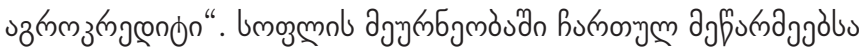

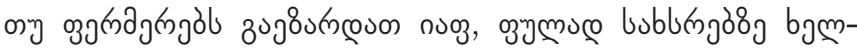

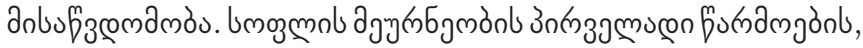

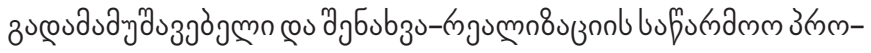

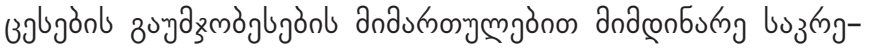

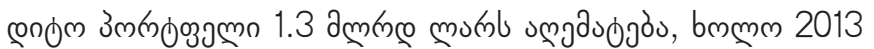

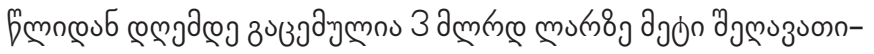

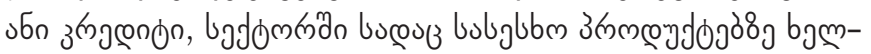

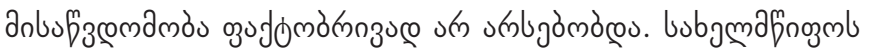

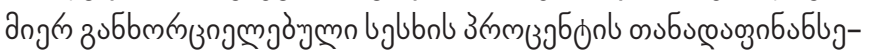

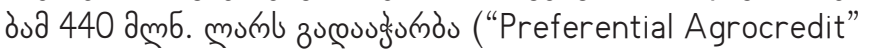
project, 2021).

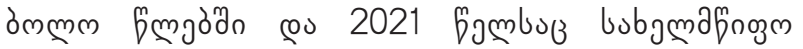

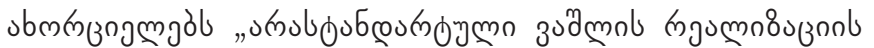

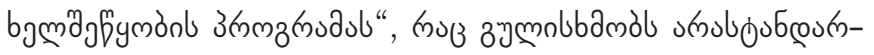

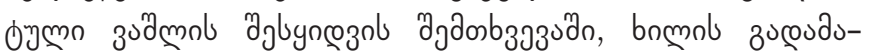

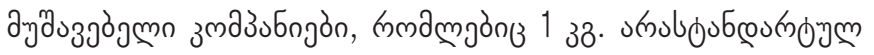

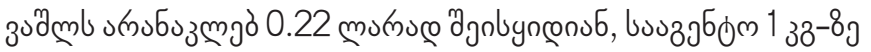
0.10 mumol megbmón byabnconol zub(zals (Industrial apple sale promotion program, 2021).

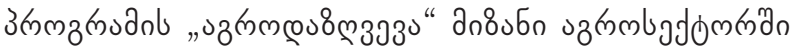

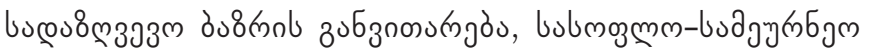

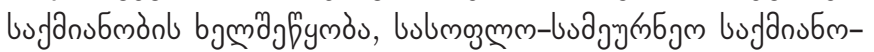

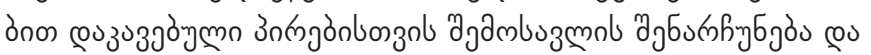

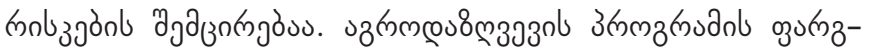

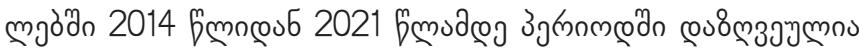

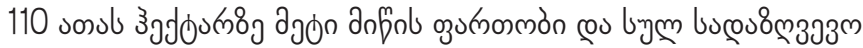

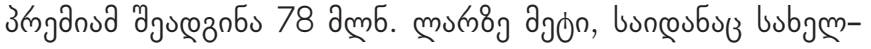

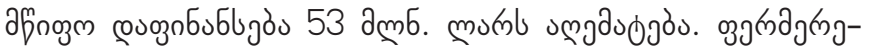

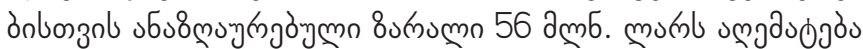
(Agroinsurance Program, 2021).

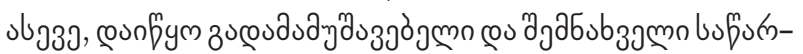

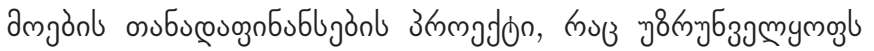

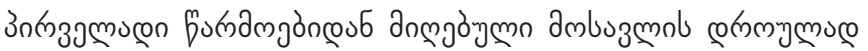

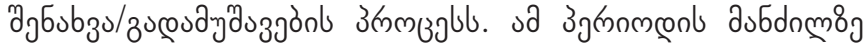

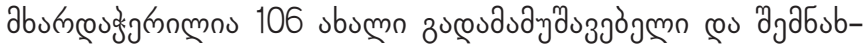

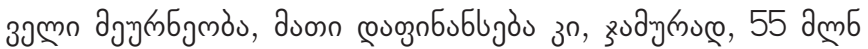
mukr uggaudjou (Co-financing of Processing and Storage Enterprises project, 2021).

ambuzmol ua

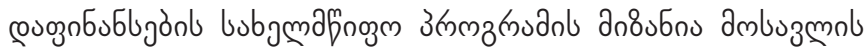

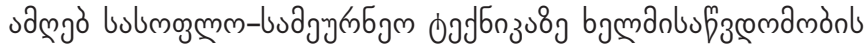

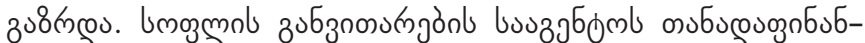

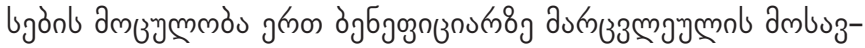

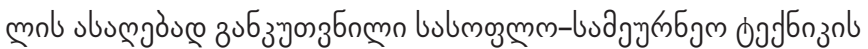

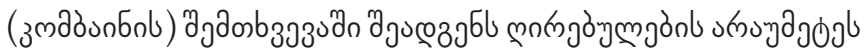

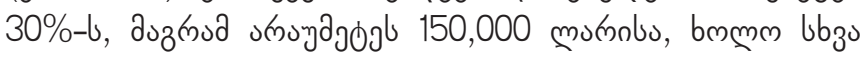

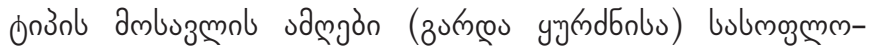

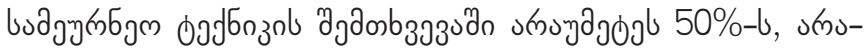
ydadogl 150,000 mumnolo (State Program for Co-financing of Harvesting Agricultural Machinery, 2021).

lmogmol agy

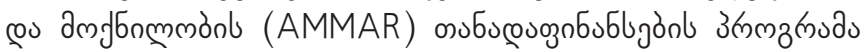

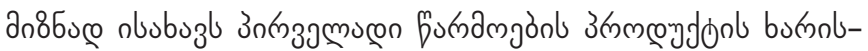

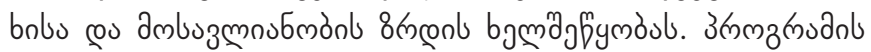

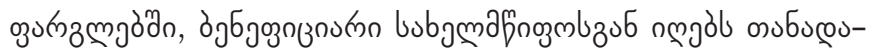

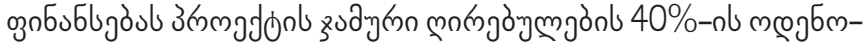

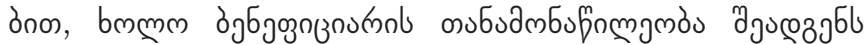
60\%-b (Agricultural Modernization, Market Access and Resilience (AMMAR) program, 2021).

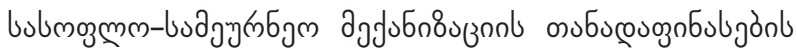

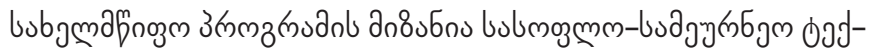

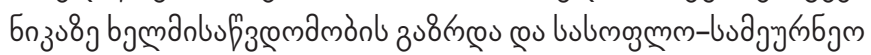

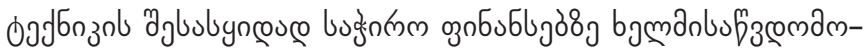

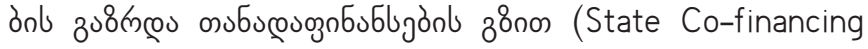
Program of Agricultural Mechanization, 2021).

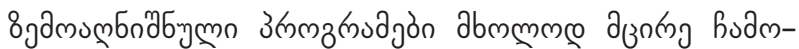

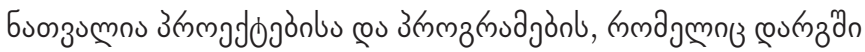

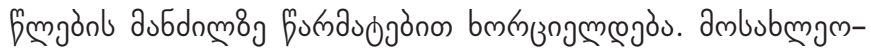
on bu bugnnm

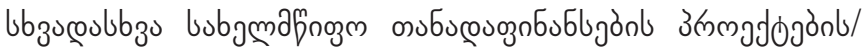

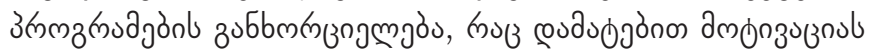

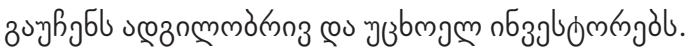

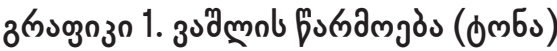

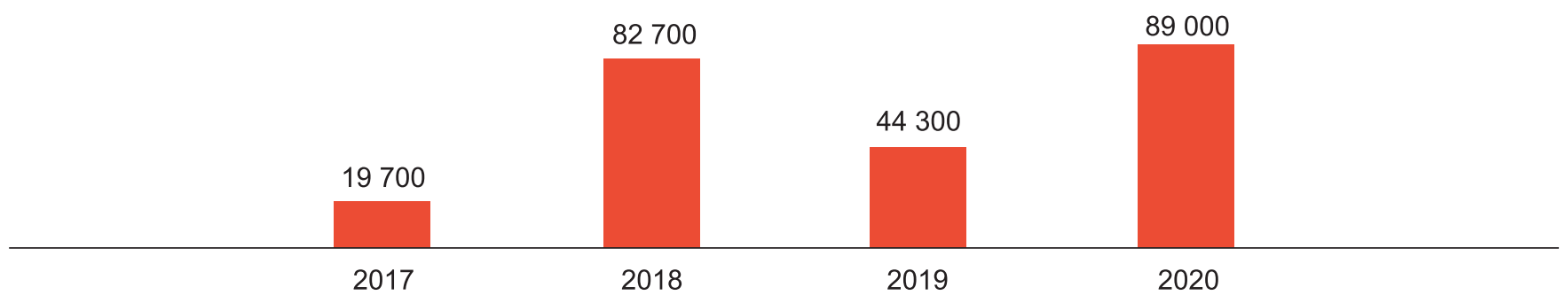

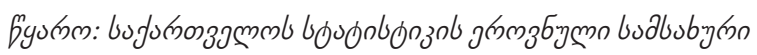




\section{9}

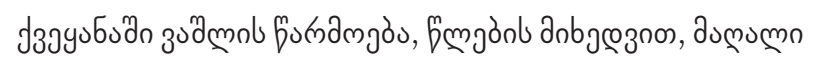

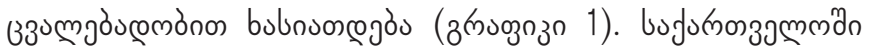

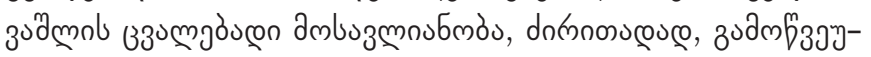

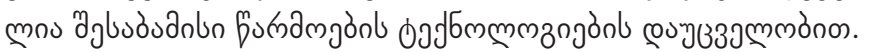

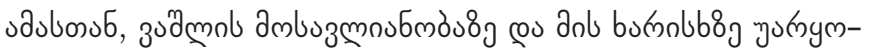

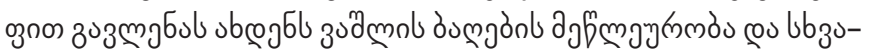

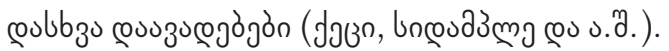

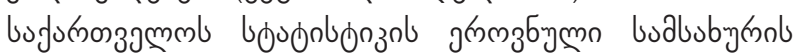

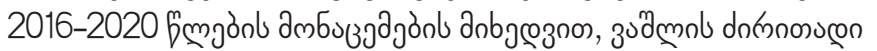

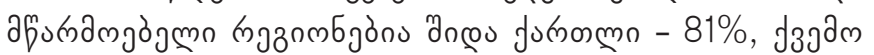

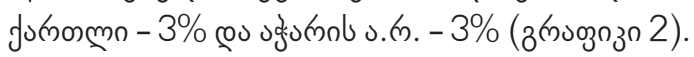

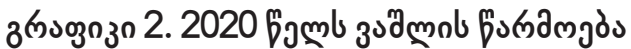

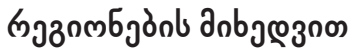

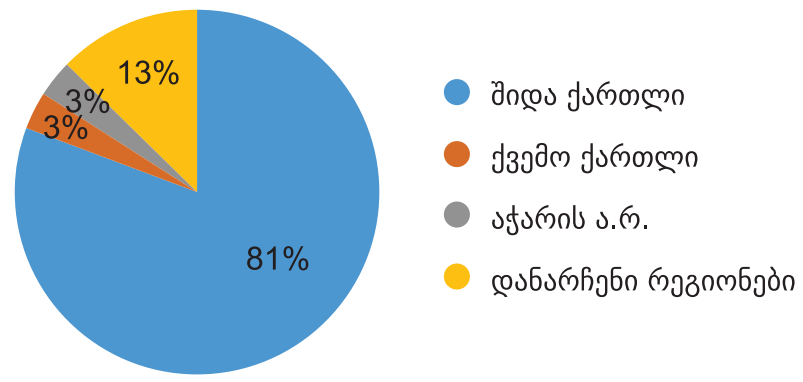

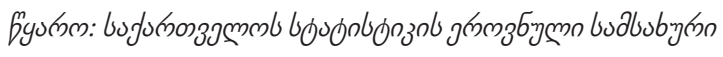

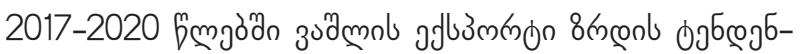

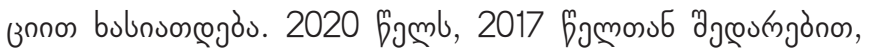

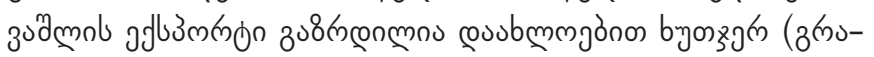

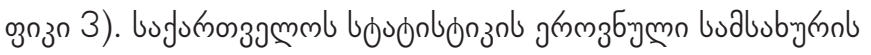

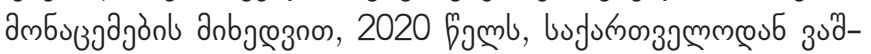

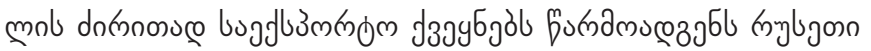

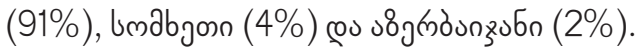

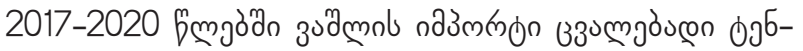

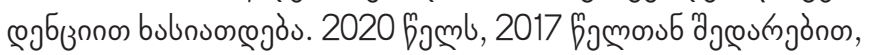

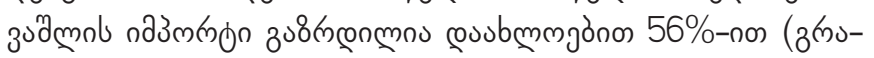

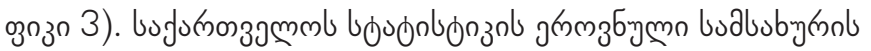

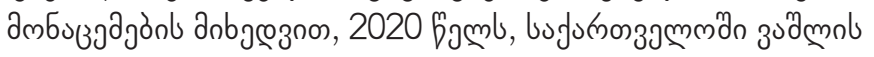

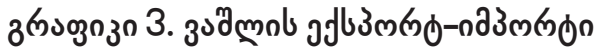

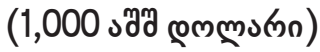

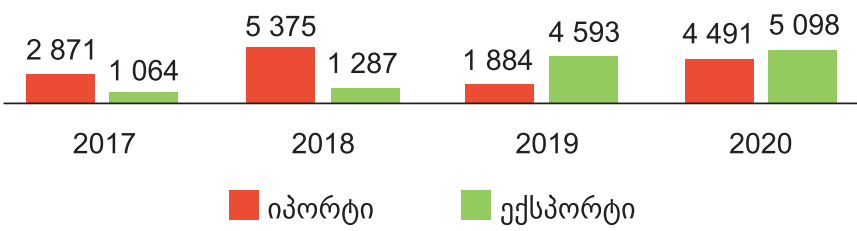

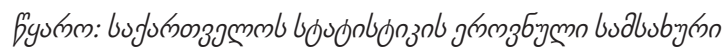

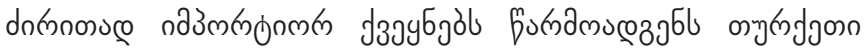

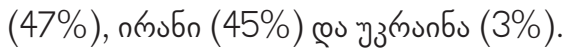

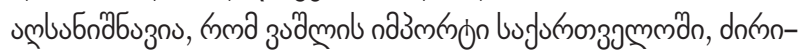

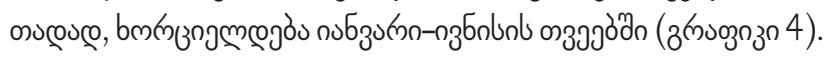

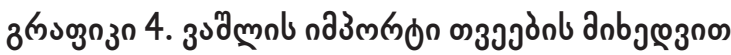

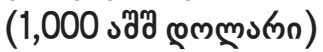

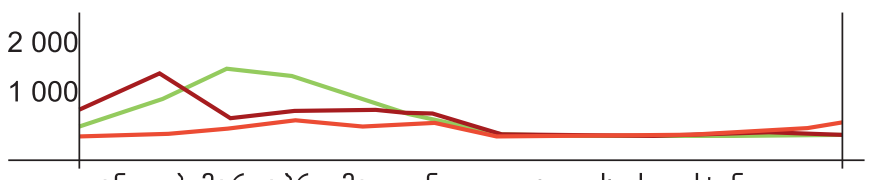

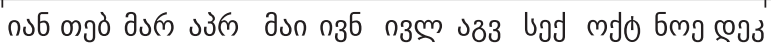
$2018=2019-20$

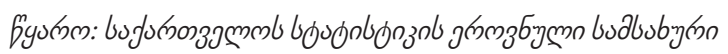

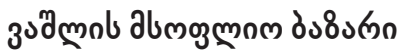

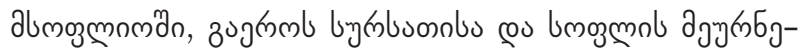

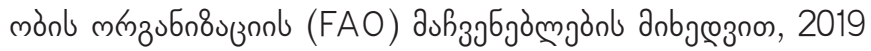

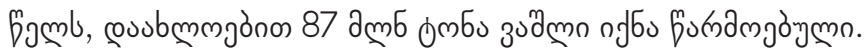

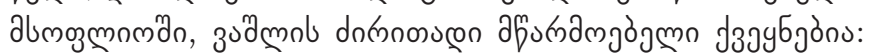

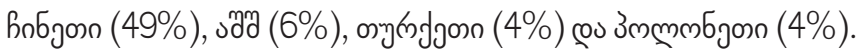

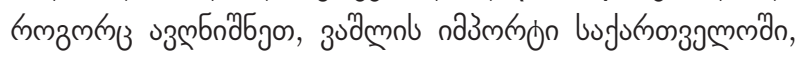

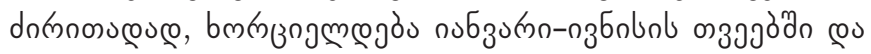

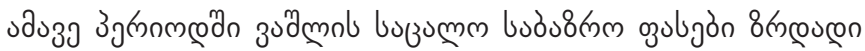
оэбе

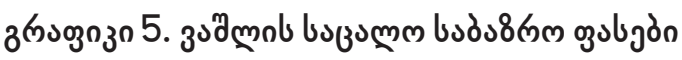

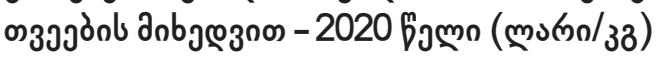

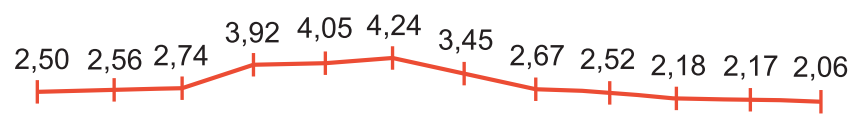

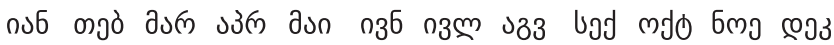

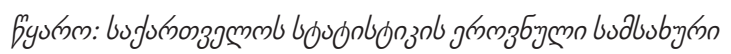

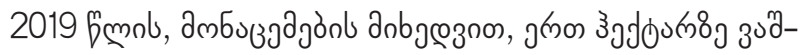

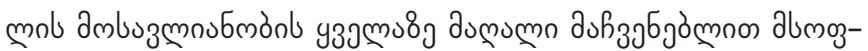

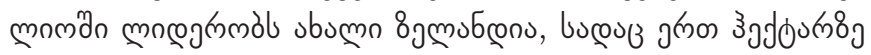

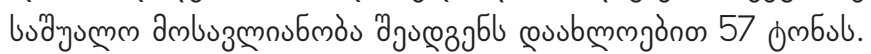

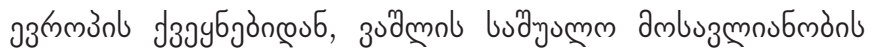

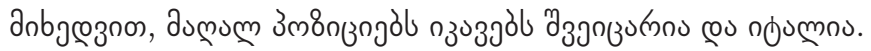

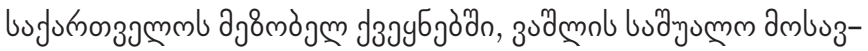

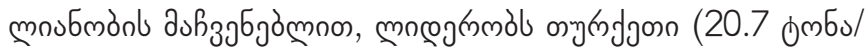

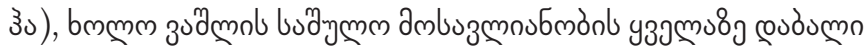




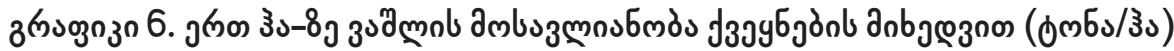

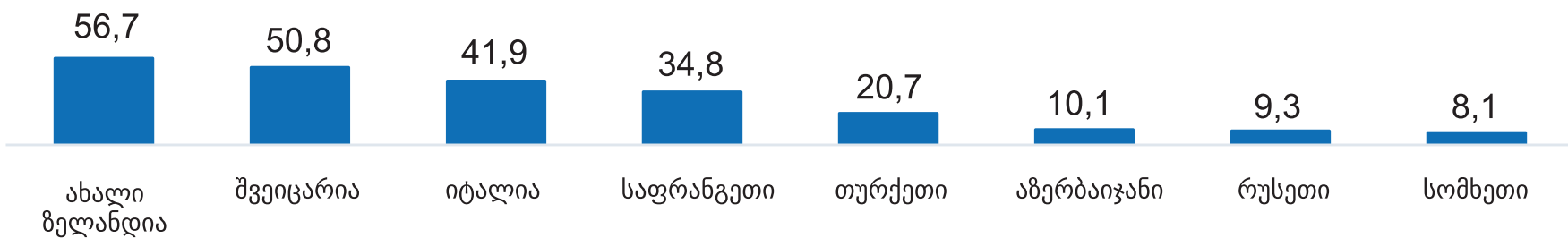

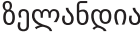

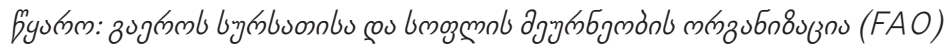

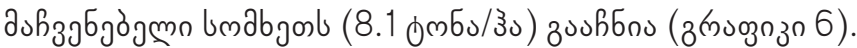

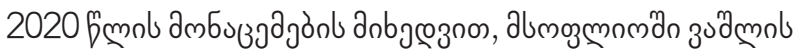

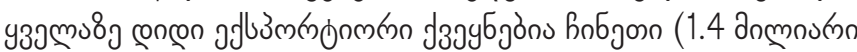

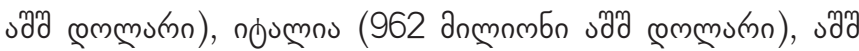

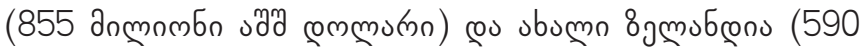

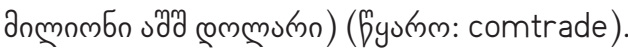

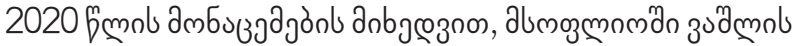

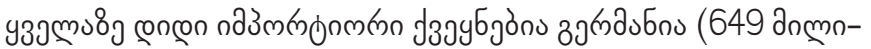

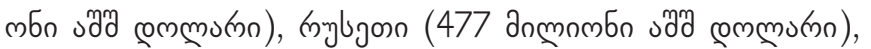
3०grnon

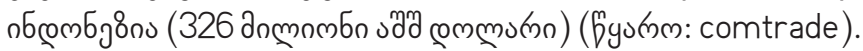

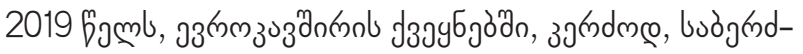

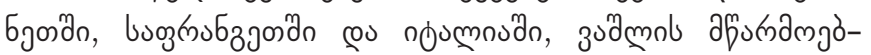

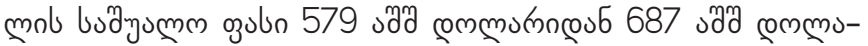

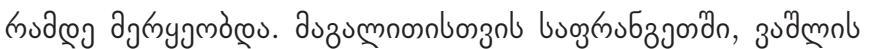

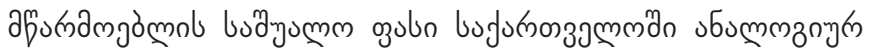

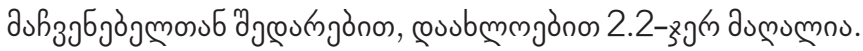

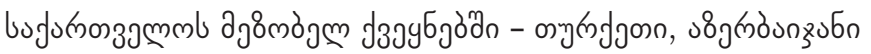

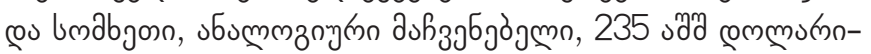

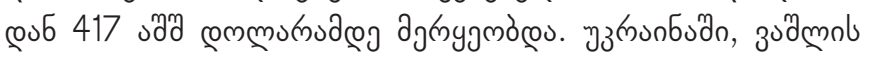

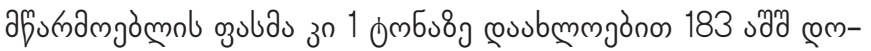

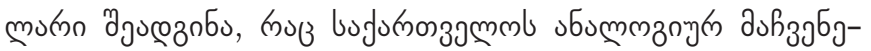

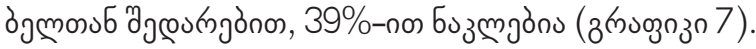

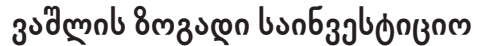

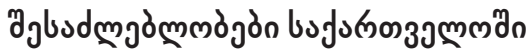

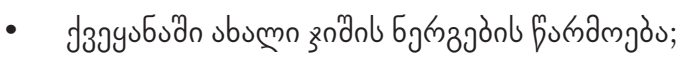

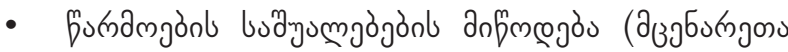
couzznb bud cos lbozo);

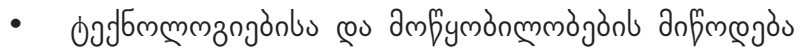

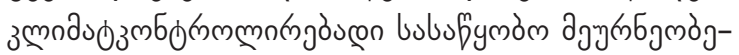
onborzol;

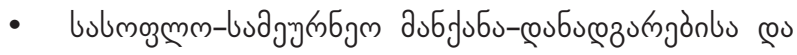

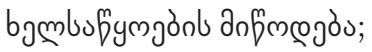

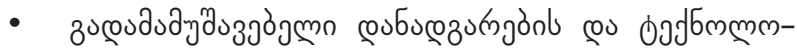

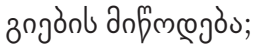

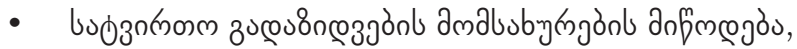

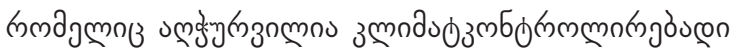
unbogajòno;

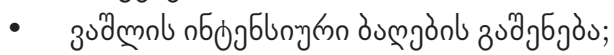

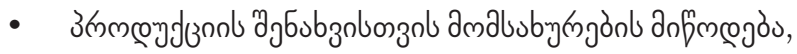

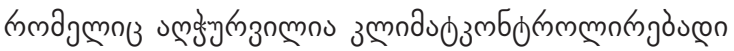
unbojajòno;

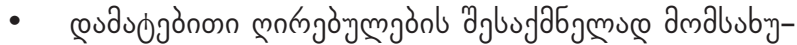

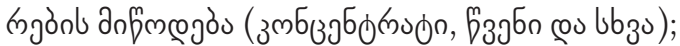

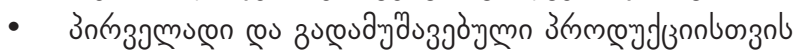

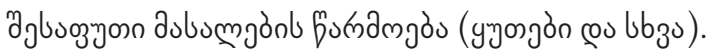

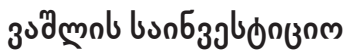

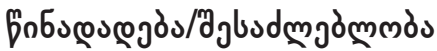

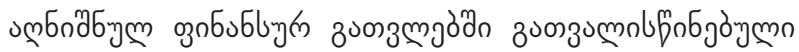

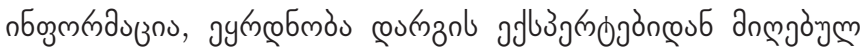

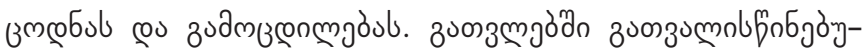

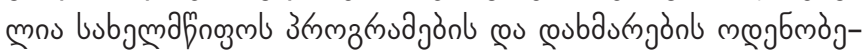

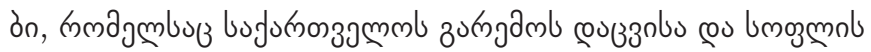

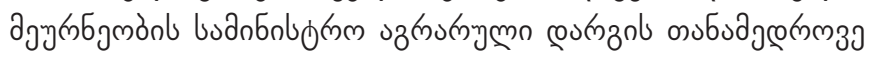

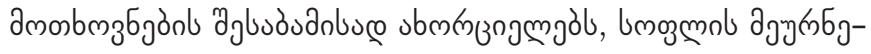

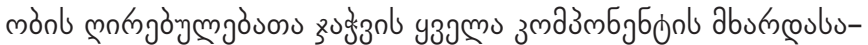

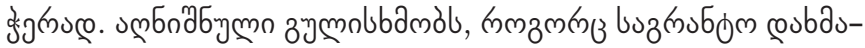

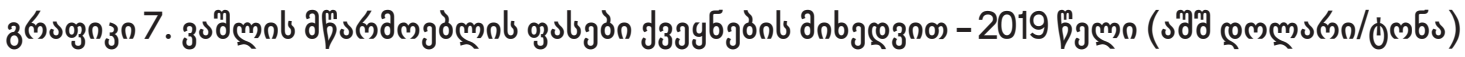

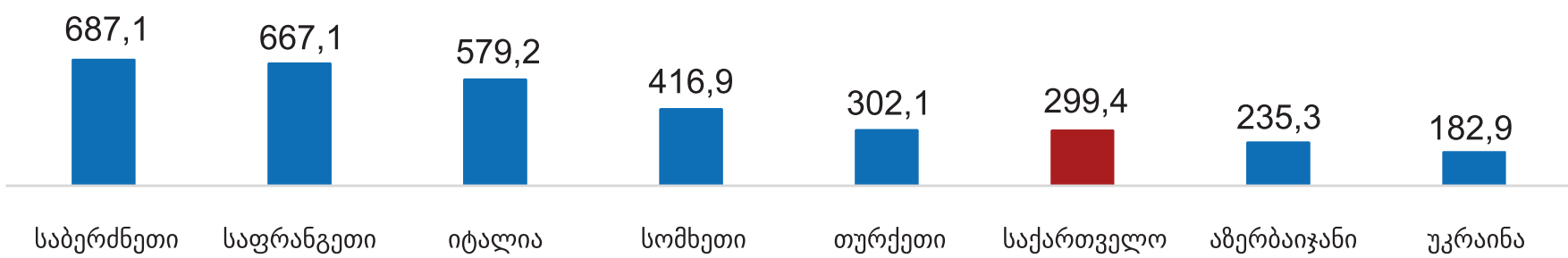

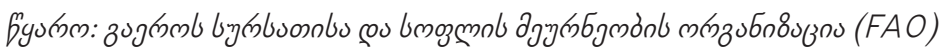




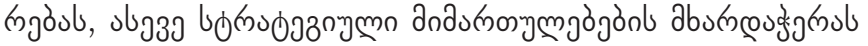

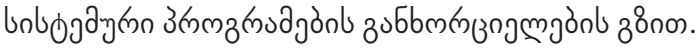

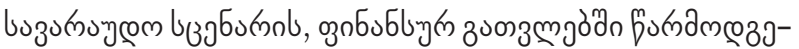

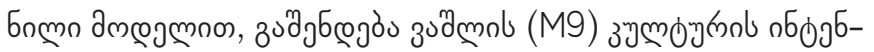

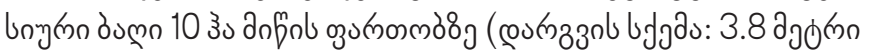
* 1 agd

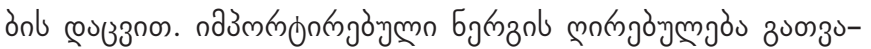

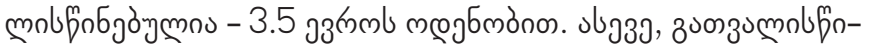

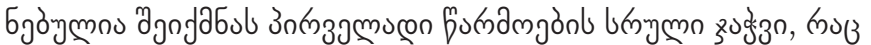

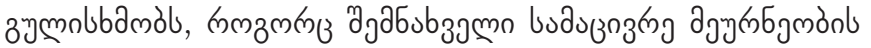

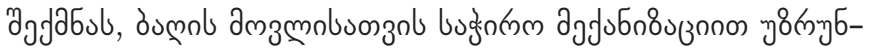

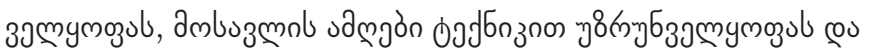

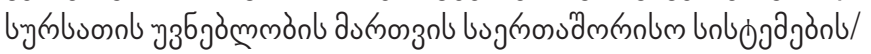

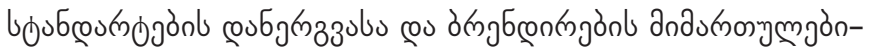

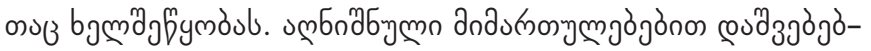

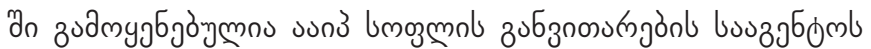

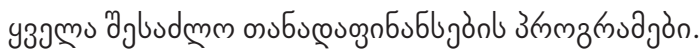

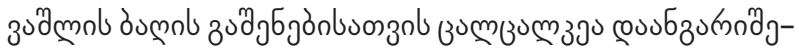

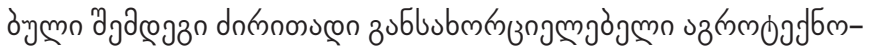

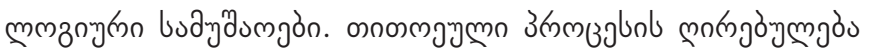

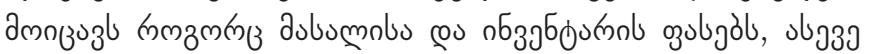

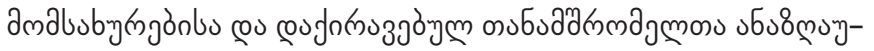

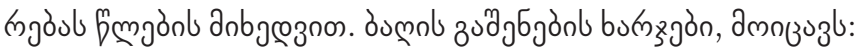

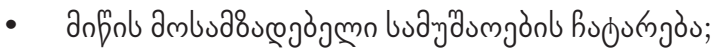

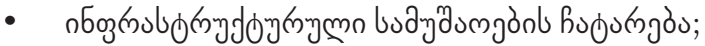

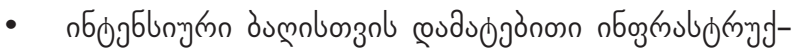
oymon a ajabs;

- Egrazarol cournzzo;

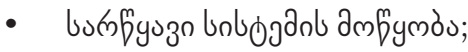

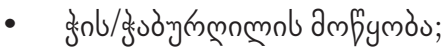

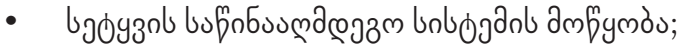

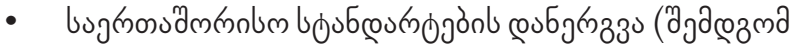

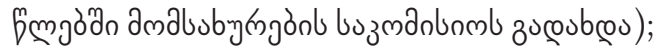

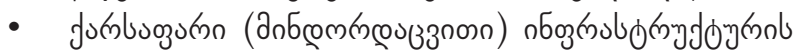

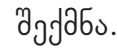

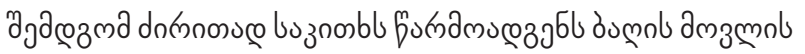

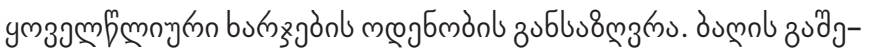

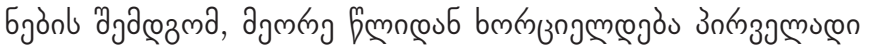

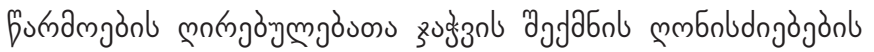

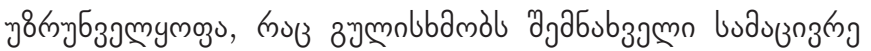

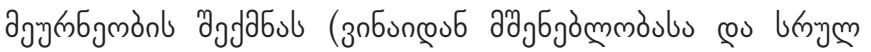

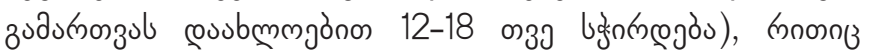

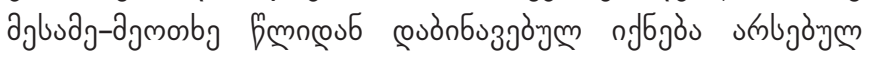

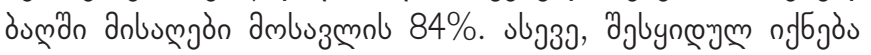

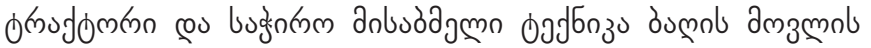

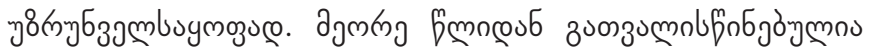

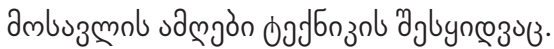

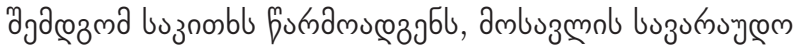

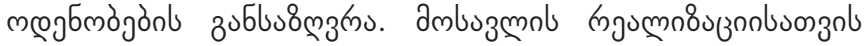

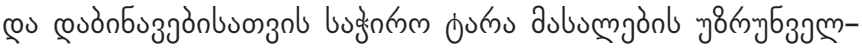

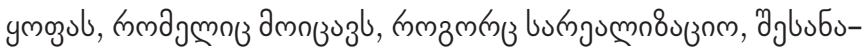

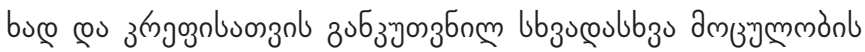

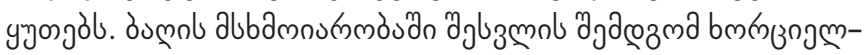

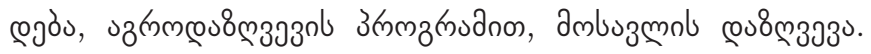

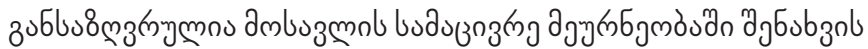

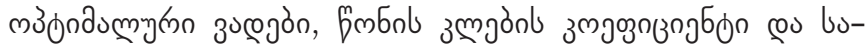
aszก弓

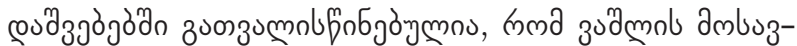

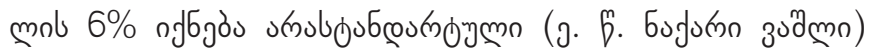

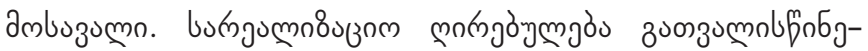

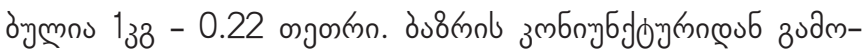

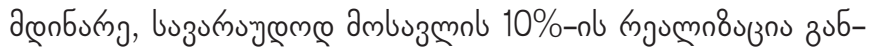

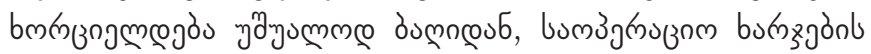

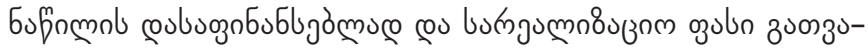

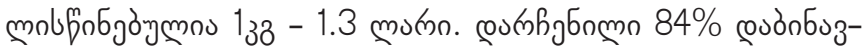

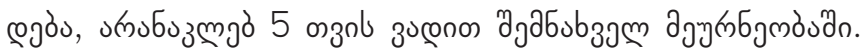

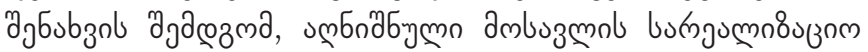

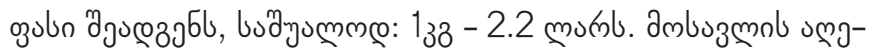

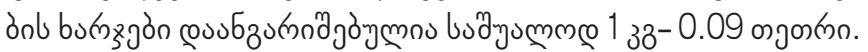

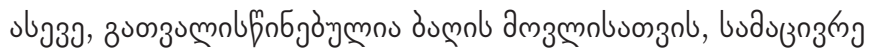

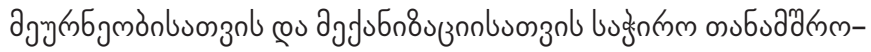

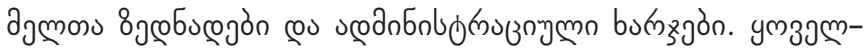

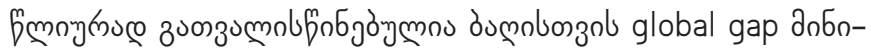

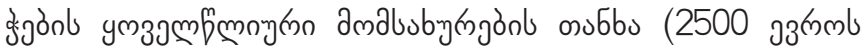

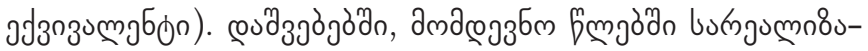

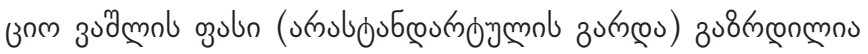

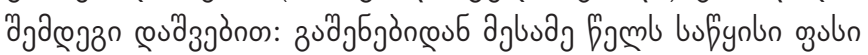

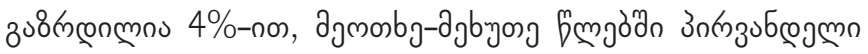

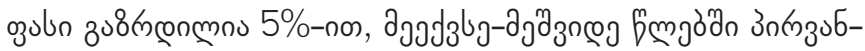

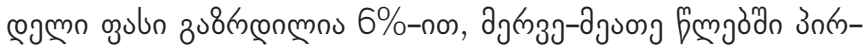

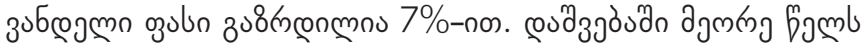

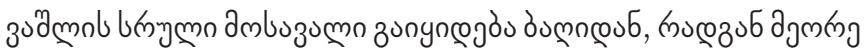

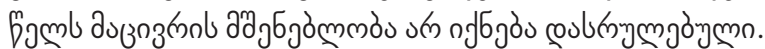

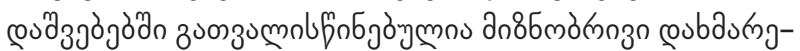

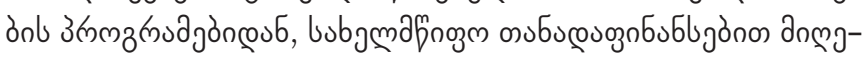

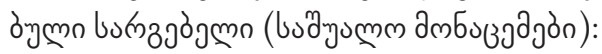

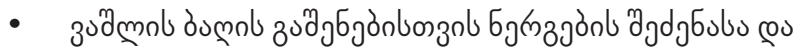

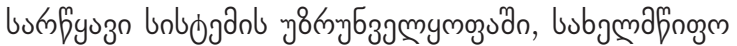

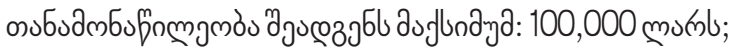

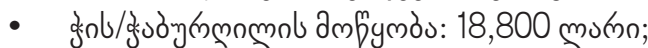

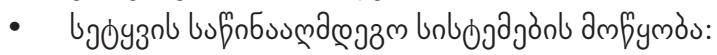

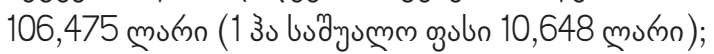

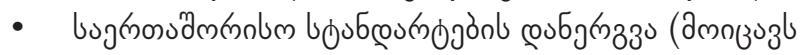

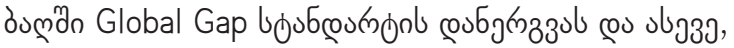

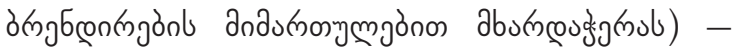
15,000 mukno;

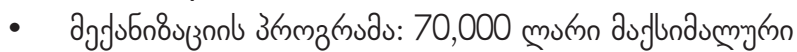




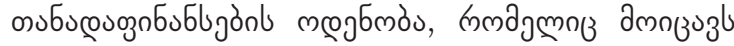

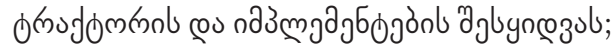

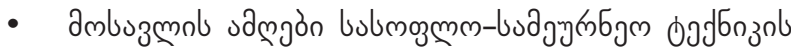

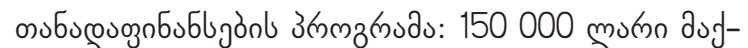

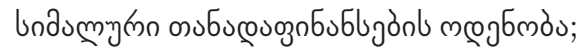

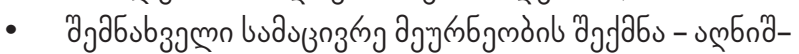

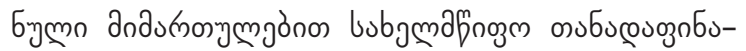

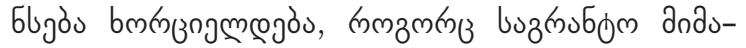

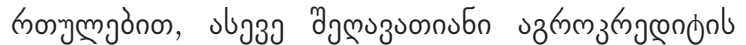

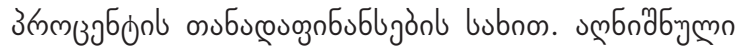

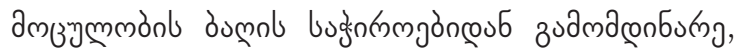

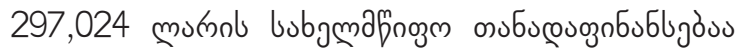

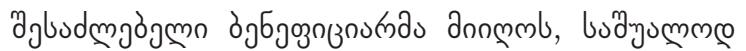

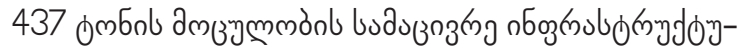
mol ajdabnbuon zol;

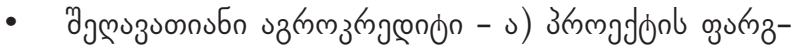

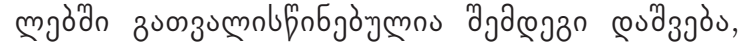

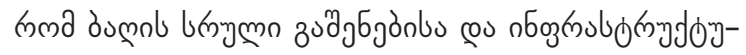

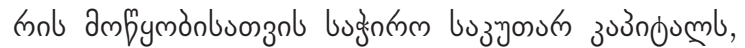

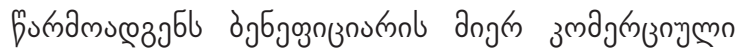

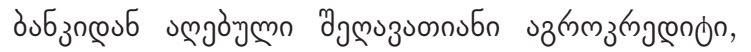

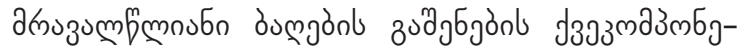

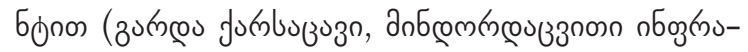

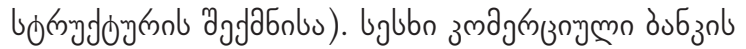

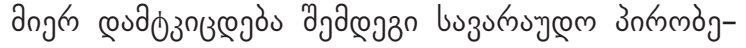

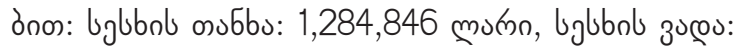

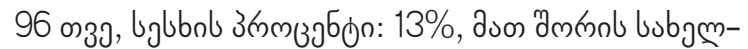

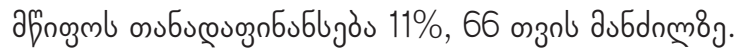

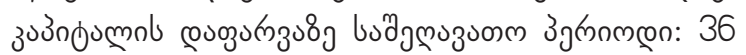

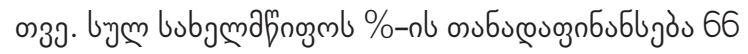

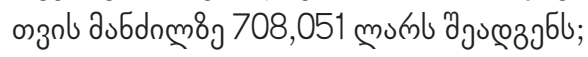

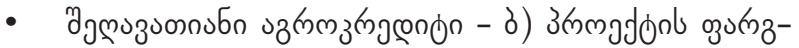

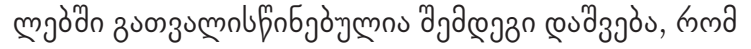

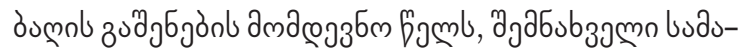

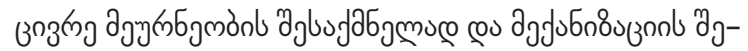

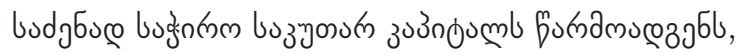

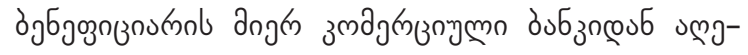

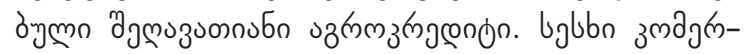

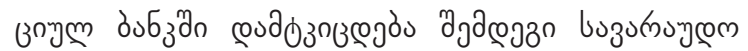

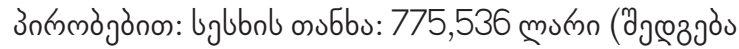

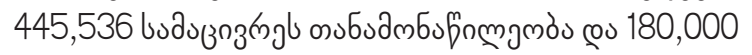

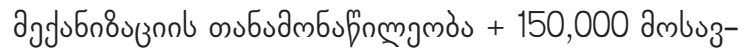

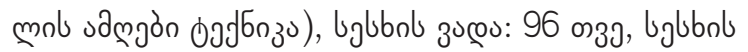

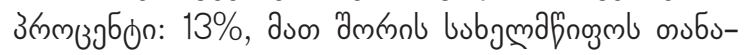

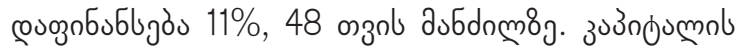

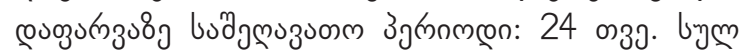

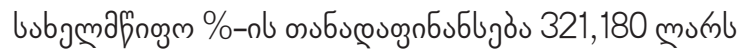
gुงевjбl;

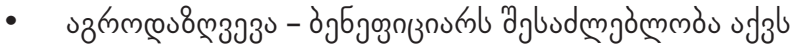

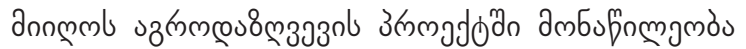

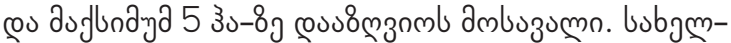

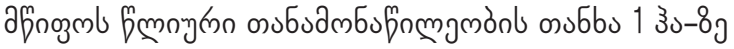

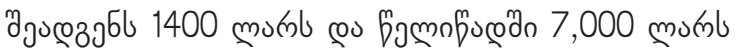

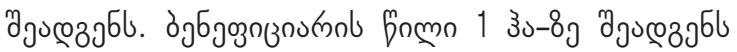
600 mumb;

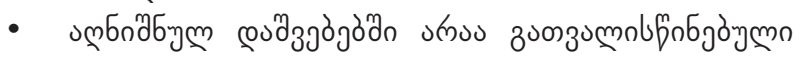

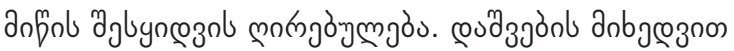

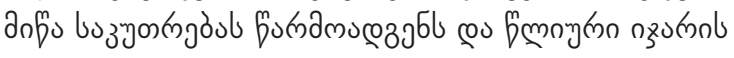

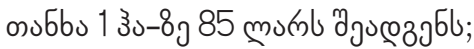

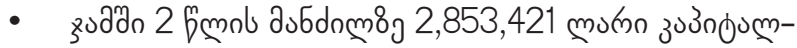

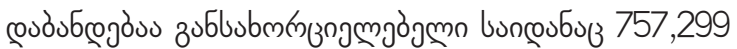

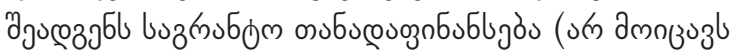

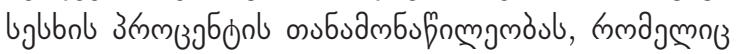

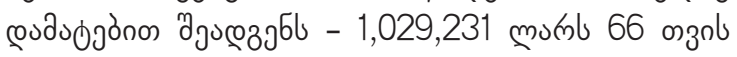

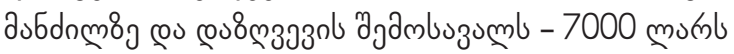

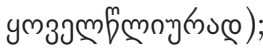

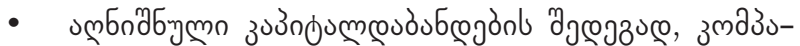

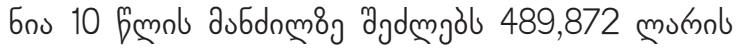

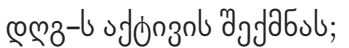

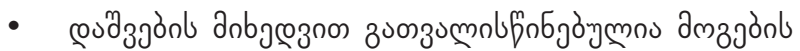

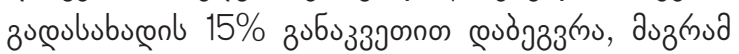

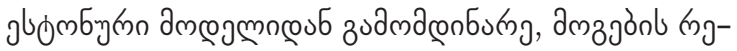

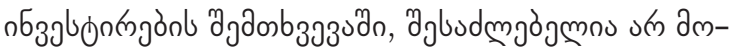

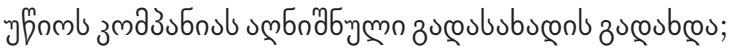

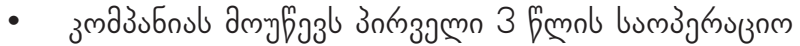

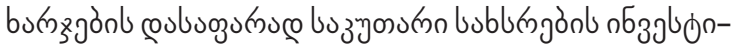

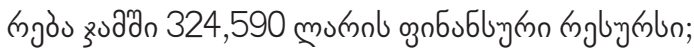

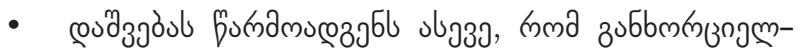

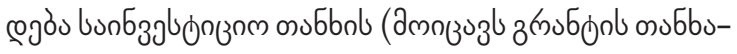

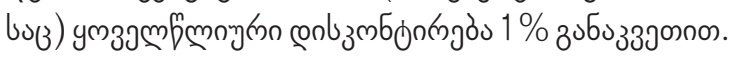

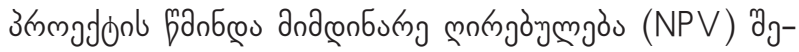

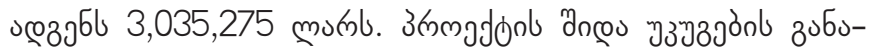

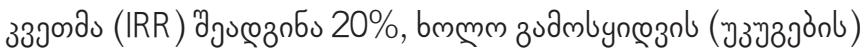

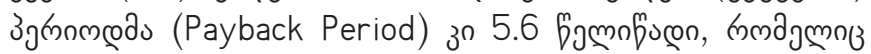

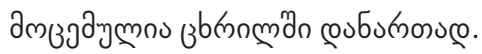

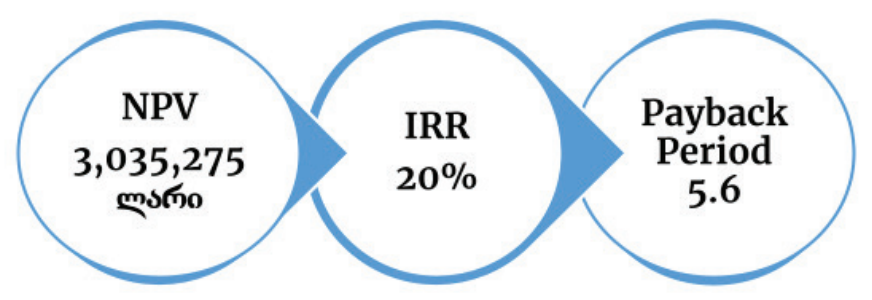

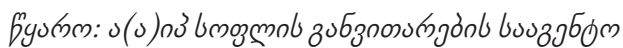




\section{3. es৬335s}

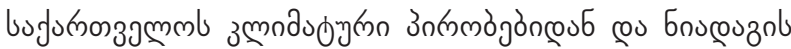

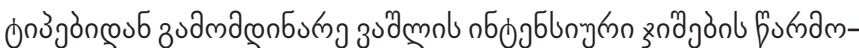

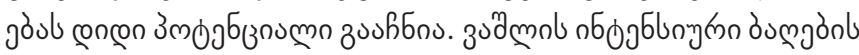

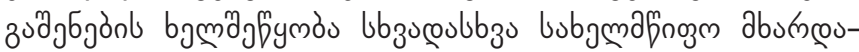

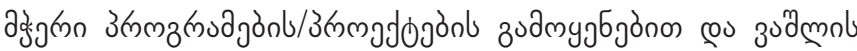

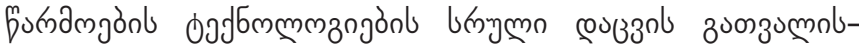

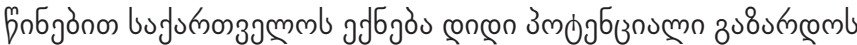

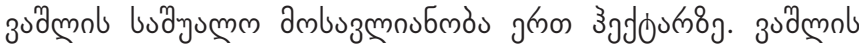

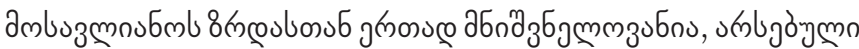

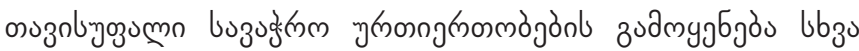

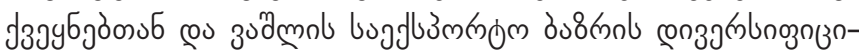

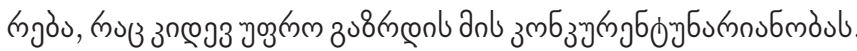

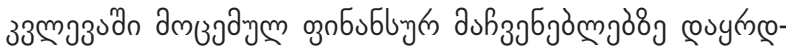

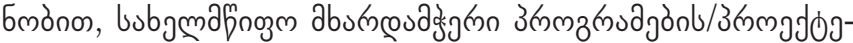

on zuдmy

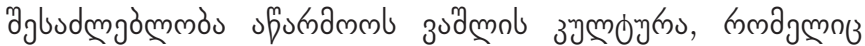

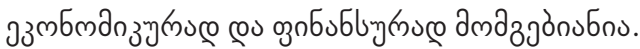

ubas,, nб

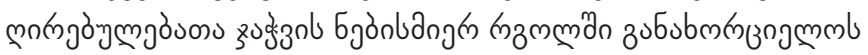

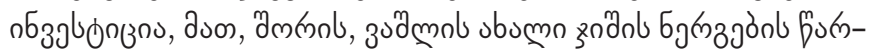

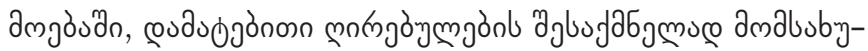

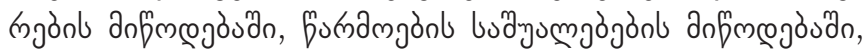

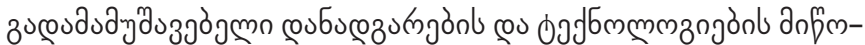
cododan cou libzu.

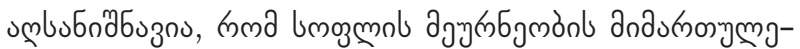

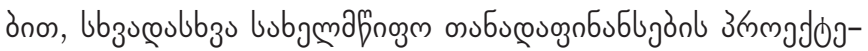
on

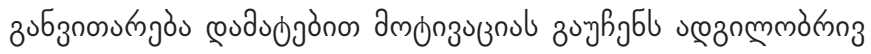

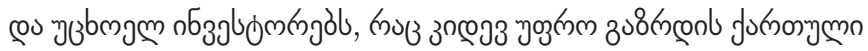
зuд

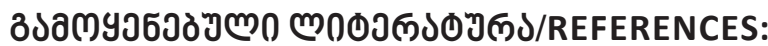

Business Registration. (2021). Public Service Hall. http://psh.gov.ge

Fraser Institute. Economic Freedom Ranking. (2021). https://www.fraserinstitute.org/economicfreedom/map?geozone=world \&page=map\&year $=2019$

Free Trade Regimes - Deep and Comprehensive Free Trade Agreement (DCFTA). (2021). https://ec.europa.eu/trade/policy/ countries-and-regions/countries/georgia/

Index of Economic Freedom. (2021). https://www.heritage.org/index/ranking

New Customs Code. (2021). https://www.rs.ge/; https://matsne.gov.ge/document/view/4598501?publication=0

NNLE Rural Development Agency. (2021). "Plant the Future" Project. https://rda.gov.ge/projects/read/plant_future/2:parent

NNLE Rural Development Agency. (2021). "Preferential Agrocredit” project. https://rda.gov.ge/projects/read/agro_credit/5:parent

NNLE Rural Development Agency. (2021). Agricultural Modernization, Market Access and Resilience (AMMAR) program. rda.gov.ge NNLE Rural Development Agency. (2021). Agroinsurance Program. https://rda.gov.ge/projects/read/agroinsurance/4:parent

NNLE Rural Development Agency. (2021). Co-financing of Processing and Storage Enterprises project. http://enterprise.rda. gov.ge/?lang=en

NNLE Rural Development Agency. (2021). Industrial apple sale promotion program. https://rda.gov.ge/projects/read/industrial_apple_\%20sale_\%20promotion_\%20program/7:parent

NNLE Rural Development Agency. (2021). State Co-financing Program of Agricultural Mechanization. http://agrotech.rda.gov.ge/ NNLE Rural Development Agency. (2021). State Program for Co-financing of Harvesting Agricultural Machinery. rda.gov.ge

Tax Code of Georgia. (2021). https://matsne.gov.ge/

Tax legislation - legal guide "Invest in Georgia". (2020). https://www.investingeorgia.org/en/

The World Bank Group. (2020). Ease of doing business in Georgia. https://www.doingbusiness.org/en/data/exploreeconomies/georgia

Zivzivadze, L., Taktakishvili, T., Zviadadze, E., \& Machavariani, G. (2021). An Evaluation of Permanent Crops: Evidence from the "Plant the Future" Project, Georgia: Open Agriculture 6(1), 212-222. https://doi.org/10.1515/opag-2021-0012 